\title{
Chronotypology: a Comparative Method For Analysing Game Time
}

Jayemanne, D.

This is the author's version of an article accepted for publication in Games and Culture (02019 Author.

Reprinted by permission of SAGE Publications. The published article is available from

https://doi.org/10.1177/1555412019845593 


\section{CHRONOTYPOLOGY: A COMPARATIVE METHOD FOR ANALYSING GAME TIME}

Author 1

ABSTRACT: This paper presents a methodology called 'chronotypology' which aims to facilitate literary studies approaches to videogames by conceptualising game temporality. The method develops a comparative approach to how videogames structure temporal experience, yielding an efficient set of terms - 'diachrony', 'synchrony' and 'unstable signifier' - through which to analyse gaming's 'heterochronia' or temporal complexity. This methodalso yields an approach to the contentious topic of videogame narrative which may particularly recommend it to literary scholars with an interest in the form. Along with some examples from conventional games, a close reading of the 'realityinspired' game Bury Me, My Love (The Pixel Hunt 2017) will serve to demonstrate the use of a chronotypological approach.

Keywords: Videogames, temporality, narrative, reality-inspired games.

Academic work on digital games benefited early from the work of literary scholars. Janet Murray's Hamlet on the Holodeck: The Future of Narrative in Cyberspace (1998), Brenda Laurel's Computers as Theatre (1993), Marie-Laure Ryan's Narrative as Virtual Reality: Immersion and Interaction in Literature and Electronic Media (2001) and N. Katharine Hayles' Writing Machines (2002), for example, are pioneering texts that derive significant inspiration from literature, cinema and theatre studies in their approaches to phenomena such as digital narratives, videogames and mixed reality.

Given these contributions, it may seem strange that the value of literary studies in relation to videogames was soon to be actively brought into question - perhaps most forcefully by Aarseth: 'Are games texts? The best reason I can think of why one would ask such a crude question is because one is a literary or semiotic theorist and wants to believe in the relevance of one's training' (Aarseth 2004, 47). These sorts of argument raised the stakes on the ontology of games and narratives. The claim, in 
its strongest form, was that texts are fundamentally different from games; therefore they require new methods that are responsive to what a game is and is not.

For these scholars, the concept of narrative served as something of a proxy for broader topics of inquiry in the human and social sciences (Murray 2005; Apperley \& Jayemanne 2012; Kryswinksa 2015). The debate on game ontology has remained largely interior to the disciplinary formation of 'game studies', but it has cast a long shadow there: a running gag among early-career researchers is that the most common opening in game studies articles is to begin with a note on the size of the videogame industry (more profitable than cinema!), closely followed by a rehash of the question of whether games are narratives or not. A mischievous nod to the long tail of this debate was given by the DiGRA 2017 conference in Melbourne, Australia, where delegates entering the conference foyer for an event found themselves confronted with two cakes: one for games and one for narrative.

\section{GAME TIME}

This author observed that most people seemed happy to help themselves to a bit of both. Although this anecdote is certainly indicative of some exasperation at a superannuated debate, a special issue on games and literary studies indicates ongoing interest. From the point of view of game or literary theory there may be something decisive about the ontology of narrative; from the point of view of literary or game studies, however, framing an inquiry in terms of narrative is just one tool in the box. It may be appropriate for some texts (a novel, an epic RPG) and less appropriate for others (a lyric poem, a puzzle game). 'Narrative' was probably just doing too much work in the original debates on game ontology - in mapping this field, Koenitz (2018) has argued that key early theorists were working with significantly different operational understandings of the term.

In fact, scholarly work on videogame narrative has been and remains strong (see inter alia Aarseth 2012; Ensslin 2014; Koenitz et al., 2014; Author 1 2018). As this special issue indicates, emerging crossovers with literary studies (and allied disciplines such as cinema, television and cultural studies) 
are opportunities to highlight different approaches and ideas. Ongoing experimentation with forms of 'interactive digital narrative' (Koenitz et al., 2014) and interactive fiction, the development of independent games with experimental aesthetics, and forays into interactivity or recursive aesthetics by major platforms that have traditionally been focused on linear media such as Netflix's Bandersnatch (2018) and Russian Doll (2019) all suggest that there is much to be gained from an interdisciplinary attitude.

Cultural institutions (such as the Victoria \& Albert Museum and the Smithsonian Institute) and independent festivals (including A-MAZE, the Independent Games Festival and Game On! El arte en juego) are also increasing the reach of aesthetically innovative digital games. Given this 'convergence culture' (Jenkins 2012), it seems likely that more phenomena will arise that are of interest to scholars working across game and literary studies. The closing case study in this paper, Bury Me, My Love (The Pixel Hunt 2017), is one such innovative work where gameplay and narrative interweave.

The salutary effect of the call for new approaches (Aarseth 2004; Frasca 2003) was a focus on videogames' medium-specificities - branching plots, interface effects, customisable characters, complex rules, among others - that are part of a game's poetics, and inflect traditional topics of inquiry for literary scholars in interesting ways. Conceptual approaches that help to articulate the emergent structures and properties of videogames, but do not overly insist on hard-and-fast differences between games and narrative can thus be useful in bridging game and literary studies. Such methods would be oriented to what Dena (2017) has termed 'sequence questions' that interrogate the ways in which points of articulation and differential structures in digital games give rise to various forms: placing the game's subsystems (which may or may not include something like narrative) in relation to one another.

A key concern of sequence questions is temporality. Videogames are a vanguard media form of 'hypermodernism' (Lipovetsky 2005), of 'time in conflict'. This is reflected in gaming's characteristic 
'heterochronia' (Nikolchina 2017), or proliferation of temporal schemes. As Knight Solaire from the highly influential game Dark Souls (From Software 2010) puts it, "The flow of time is convoluted". In terms of analysis, it seems very difficult to come up with a unit of game time that would be a satisfactory equivalent of those that are very useful in argumentation about literature or cinema: something like pagination or a timestamp. Heterochronia is reflected in the highly involved and selfreflexive discourse of game enthusiasts, which often involves complex grammatical negotiations of tense and person (Vught and Schott 2012).

A method for describing temporal structures in games, then, can help identify game structures of interest to a given research project, and thereby add specificity and precision to interdisciplinary analyses. This paper outlines the method of 'chronotypology' as just such a comparative approach to videogame time. Originally developed in the context of a broader theory of performativity in digital games (Author 1), here the ideas will be streamlined to specifically focus on time. As a theory of temporality, chronotypology provides a way of discussing storytelling, narrative, theme and character as they emerge through videogame performances, without overly entangling with the complications of ontological and theoretical debates around what a videogame is or is not. While this method is not primarily focused on narrative, a useful conceptualisation of narrative does arise from the terms used.

This approach may be of particular interest to literary scholars because it is efficient, utilising only three terms - two of which, diachrony and synchrony, will already be familiar to many literary scholars (although here the usage is quite specific). The third term, unstable signifier, underpins the comparative approach, referring to a particularly intensive temporal framing device. This lightweight terminology allows critics to get on with their critique of navigable texts such as videogames without too much in the way of theoretical preamble. This paper will first outline the benefits of a comparative method which draws game and literary studies into proximity. Second, the method will be outlined along with some brief examples. Finally, a chronotypological analysis of the 'reality inspired' game Bury Me, My Love will serve to illustrate the method in detail. 


\section{CHRONOTYPOLOGY AND COMPARATIVE METHOD}

Narrative design is one area in which the heterochronic complexity of game time is particularly legible. This can be discerned in narrative professionals' accounts of their work and the exigencies of the wider development context. Developing both linear and conditional experiences entails conversance with the wide variety of systems that may comprise a digital game. In some cases, the work of narrative designers and writers may need to be stretched across demands from other disciplines such as art, programming and design. They may be brought on late in a development cycle to provide 'narrative triage' or work as a script doctor (Skolnick 2014, 104-106) - the assumption being that narrative is quite amorphous and capable of fitting around the structures mandated by gameplay, level design and so on. This attitude is reflected (and lampooned) in Bissell and Burns' The Writer Will Do Something (2015), where a hapless writer must navigate the contradictory production logics that come to a head in an emergency production meeting with the team leads of the fictional game ShatterGate: Future Perfect. The final punchline is that the only person less important than the writer is the audio department, who weren't even represented at the meeting in the first place.

Heterochronia is also reflected in the tools that writers and narrative designers use. Despain explains that there is a lack of specialist and industry-standard software or workflows for game writers (although there may well be bespoke tools built for a particular studio or game). Instead, she notes the prevalence of general software tools such as Excel, which are 'not likely to be joined there on the shelf by a box with a program specifically for game writing any time soon. If such a program were made flexible enough to work with every game genre in existence, it would look pretty much like Excel' $(2008,18)$. The software tools optimised for organising narrative alongside other temporal forms in videogames would be very similar to the tools designed for organising modern things in general.

This suggests that the heterochronias of everyday life and digital games reflect one another at a deep level. Ryan (2014) argues that the abstractness of time raises significant difficulties for theoretical discussions of interactive narrative. Where spatiality is 'widely considered a distinctive property of 
digital media... their temporality has attracted little critical attention... digital media play with time as much as they play with space' (118). This is because 'digital media are much more efficient at giving us the experience of space than at intensifying our awareness of time' because '...time is much more abstract than space'.

The difficulties of analysing abstract digital game time are also recognised in Zagal \& Mateas' (2010) paper on game time. Critiquing the 'Platonic' conception of time as an empty unit in which events occur, Zagal \& Mateas instead argue for a method that is 'relational'. In place of time conceptualised as an accumulation of abstract units, Zagal \& Mateas argue for the analysis of events and the way that these relationally produce 'temporal frames'.

Zagal \& Mateas' 'relational' approach has resonances with comparative modes in the study of literature. In their introductory text, Dominguez et al (2015) describe how comparative literary studies can be an exciting discovery for students in which 'one realises there is a big world beyond the limits of the "national" literature that has provided the sheer bulk of compulsory readings during primary and secondary school' as comparative approaches open onto 'another form of reading - neither better, nor worse, just different' (ix). Much like this view of comparative literary studies, chronotypology offers another form of reading that sidesteps the ontological debates internal to game studies to provide a way of relating the literary and ludic which is neither better nor worse than ontological accounts; just different.

Dominguez et al. assert that the comparativist move of juxtaposing putatively stable objects of study can often lead to questions about whether those objects are so stable as was first thought. A national literature may seem 'a common-sense notion', but here comparative literature poses 'challenging questions. If comparison is a matter of reading across linguistic borders, what counts as a language? Is twentieth-century English or Spanish a different language from seventeenth-century English or Spanish?' (xii). Comparative literary scholarship can often lead not to certainties about distinct 
objects of study but to 'An acute awareness about literatures influencing each other'. Similarly, each 'national' culture gives rise to comparative historical questions, as famously fictionalised by Borges by making Pierre Menard the author of Don Quixote. What starts with the neat comparison of units instead ends up pointing to integral variegations and connections that complicate classificatory schemas.

Zagal \& Mateas' recommendation of relational methods and critique of abstract notions of time align with this perspective. They develop four 'temporal frames' for analysing game time, which include Real world time; Gameworld time (referring to events that take place within represented space); Coordination time (the relation between the actions of multiple agents, or human and A.I. agents; finally, Fictive time, which is 'established through the application of sociocultural labels to a subset of events' (9). However, as the authors admit, beyond these four 'different temporal frames can (and should) be defined' (16) - but the method for doing this remains somewhat ad hoc.

\section{CHRONOTYPOLOGY: DIACHRONY, SYNCHRONY, AND UNSTABLE SIGNIFIERS}

Chronotypology provides a way forward by building from Giorgio Agamben's essay on play, ritual and temporality "In Playland" (which itself complicates work by Benveniste and Levi-Strauss). The method comprises three key terms: diachrony, synchrony and unstable signifier. Diachrony and synchrony comprise two inverse temporal movements. Synchrony is associated by Levi-Strauss with ritual and, in recreating a sacred event or drama from the past within the present, draws temporal frames together. An occidental example would be a holiday such as Christmas, where nativity scenes re-create or synchronise the past within the lived experience of the present.

Agamben supplements these ideas by elaborating a concept of diachrony which, significantly, is associated with play. If synchrony brings timeframes together, diachrony splits them apart, as in Pinnocchio where 'Playland' represents an eternal holiday without the structuring principles of the calendar: time seems 'out of joint', to whirl past. Many a gamer, glancing in horror at a clock after a 
particularly immersive session of play, can attest to the capacity of digital games to generate this sort of experience.

Although this may seem like a binary opposition, in reality neither diachrony nor synchrony can complete their signifying operation. There is always a material remainder. In the context of synchrony, Levi-Strauss notes that the sacred object must be carefully hidden away once the ritual ends. For Agamben, the toy presents an analogous — but opposite — transformation when it leaves its own space:

...the toy, as a representation of a pure temporal level, is undoubtedly a signifier of absolute diachrony, of the prior transformation of a structure into an event. But here too this signifier, once freed, becomes unstable, and is invested with a contrary meaning; here too, at the end of the game, the toy turns around into its opposite and is presented as the synchronic residue which the game can no longer eliminate. (Agamben 1993, 79-80)

There is thus a constitutive tension between diachrony and synchrony: 'the pure event (absolute diachrony) and the pure structure (absolute synchrony) do not exist' (70). Diachrony and synchrony are tendencies and not essences. Temporal signifiers are liable to flip valency, as shown by the limit cases of the toy and the ritual object respectively. Agamben terms these 'unstable signifiers'.

The association of play with diachrony may suggest that videogames are basically oriented towards this tendency - and game culture is certainly obsessed with novelty and change. Levi-Strauss' association of synchronic ritual with seasonal, initiatory or cosmic timeframes would further tend to suggest that synchrony has little to do with digital games. However, videogames are capable of generating powerful synchronic experience: rhythms (Apperley 2010), repetitions (Nikolchina 2017) and interminable aporias where players find themselves 'stuck' and unable to proceed (Aarseth 1997). Every game of Fortnite (Epic Games 2018), for example, will start with players flying above a standard map. Every death or fireside rest in Dark Souls resets the world's enemies to their original 
positions. The Save Game synchronises a current game state as a point from which to depart on future diachronic explorations of the game's possibility space.

Similarly, although Agamben associates the concept of the unstable signifier with objects whose materiality belies the very semiotic-temporal relation they exemplify, unstable signifiers in games may not be 'material' in the narrow sense - as anyone who has stepped on a Lego piece in the dark can attest, contemporary cultures can exhibit a bit less anxiety about stashing away toys. Instead, computers in particular allow these signifiers to proliferate. As Ryan notes, in digital culture, 'the computer has certainly done much more for our use of time than for our actual mobility through space... However, we tend to take these services for granted, and whenever the computer makes us think about time, it is because it frustrates us with lag and slow downloads' $(2014,118)$. The unstable signifier can be one of the most potent ways of registering temporal structure - as in the glitch which crashes the game, and makes us realise how long we've been playing.

Not all examples need be so drastic. Computers are capable of presenting myriad signs, all of which can very easily be assigned new values and hence constitute a type of 'unstable signifier': a quantitative shift which leads to the qualitative capabilities of digital games. With these adjustments in perspective, Agamben's “In Playland" provides the materials for an efficient and versatile method of analyzing game heterochronia. Chronotypology consists of accounting for the diachronic (separation of timeframes) and synchronic (convergence of timeframes) dynamics of a given game text, element or action. Unstable signifiers are capable of changing and switching between these tendencies.

One of the simplest - but most instructive - examples is a cutscene (all players will experience this timeframe, synchronizing their experiences) followed by a binary decision (players must choose one option, splitting the game into one of two diachronic timeframes). In another example, the remake of Resident Evil 2 (2019) generates both synchronic nostalgia for the previous game (character and level 
design) while at the same time insisting on the diachronic changes in game technology (graphics, audio, smoother U.I. and voice acting).

The realities of game development mean that wildly propagating possibilities (such as those resulting from iterated branching structures) can be difficult to build and implement fully. It is often a design imperative to modulate and control diachrony - or more precisely, to imbue highly synchronic structures with a sense of diachrony. This leads to what Ford terms the 'hourglass story shape' (2016, 25 ) in which players may be given the sense of a highly diachronic story space, which is in fact structured such that it inevitably ends up in a predetermined synchronic nodal or ending point. Terms such as 'critpath' (Heussner et al., 2014) designate the mandatory - and hence synchronising - goals that players must complete in order to advance in a game.

These synchronic design structures highlight the importance of Agamben's discussion of limit cases and the 'end of the game'. This is the point at which the limits of play become particularly instructive for the comparative analysis of narrative in navigable texts such as videogames. Players approach the weft and warp of temporal experience in gaming by attempting to discern what signifiers are unstable and will, as the game proceeds, change their temporal signification.

Play proceeds from the will-be-played (the highly diachronic perspective of the neophyte for whom everything appears as a potential unstable signifier) to the can-be-played-with (a balance of diachrony and synchrony in the mature play experience) and finally the willalways-be-played (the endgame, where the most signifiers have become stable and highly synchronic). (Author 1)

Narrative, in this comparative view, can be defined as a highly synchronising element in those games with strong storylines: in spite of various differences in how players conduct themselves in the ludic 
portions of the game, narrative draws the various individual performances together through certain events that will occur in every playthrough.

However, this synchrony is only perceptible for players at the end of the game. In the course of play, narrative is experienced in highly diachronic terms. As a game's storyline progresses, players anticipate what signifiers are unstable (in both ludic and narrative senses), allowing them to actively intuit the synchronic conditions that will obtain at the game's conclusion once its diachronic possibilities are exhausted or oversaturated. This is what Costikyan terms 'narrative anticipation' $(2015,94)$, which can be seen as a game design analogue of Jauss \& Benzinger's 'horizon of expectation' (1970). A key example is Gone Home (2013). In this game, the design initially creates a strong sense of narrative anticipation through the tropes of horror gaming, only to subvert this in subsequent gameplay and narrative developments. The game thereby introduces a new sense of comparative diachronic experience which conditions the sense of synchrony that it initially works to set up.

Conversely, the synchronic aspect of linear game narratives, hidden or dissembled in a first playthrough, becomes highly evident upon replay. No matter what gameplay options or builds a player chooses, in the 'New Game+' mode of God of War (2018), player character Kratos will always return to his home to retrieve the weapons which symbolize his sordid past - players have no influence on this event through play. The recurrence of the same narrative beats or cutscenes eliminates narrative anticipation, even as different ludic possibilities may be explored.

\section{"BURY ME, MY LOVE” AND THE 'STANDARD METAGAME'}

In this way, chronotypology can aid in nuanced analysis of conventional game design types (the original book treatment (Author 1) contains a wider range of in-depth examples). In concluding this paper, I will turn to a unique game in order to further demonstrate the versatility of the method's approach to time. 
Nour is the fictional deuteragonist of Bury Me, My Love (BMML), a mobile-based game by French creators The Pixel Hunt. Players take on the role of Majd, Nour's husband. Majd, the player character, is looking after ailing family members in the war-torn Syrian city of Homs and is unable to accompany his wife Nour as she leaves to seek refuge in Europe. The pair have invested in smartphones in order to keep in touch, and Majd follows his wife's journey through instant messages in the style of WhatsApp.

Along with the subject matter, which presents ethical quandaries and issues not often dealt with in gaming, BMML incorporates itself into the everyday rhythms of smartphone use: in its 'real-time' mode, the game consists of replying to Nour's 'instant messages' which pop up with notifications like any other app on your phone. As Majd, you must remotely interact with Nour much as you would with a real interlocutor, sending texts, emojis and selfies to advise, cajole and reassure.

Nour is presented as a free-spirited person however, and much of the game's effectiveness arises from this portrayal: players can never be quite sure if she will follow their advice. One of the game's creators, former journalist Florent Maurin, has been careful to distinguish $B M M L$ from a game which is simply 'about' a certain issue or event in the news. Instead, when thinking about a possible generic classification for his game, Maurin offers the term 'reality-inspired'. As McMillan elaborates, 'Reality-inspired Games (RIG's) incorporate genuine stories from the perspective of a few or encompasses the values of a larger section of those affected by a specific issue' (personal correspondence, 2018). Rather than utilizing reportage to guide its design response to such an ongoing and significant event as the refugee crisis in Europe, $B M M L$ pursues a different strategy. Through condensing and adapting to multiple stories, the RIG makes game design part of a journalistic and documentary process that lands somewhere between fiction and non-fiction.

In terms of interface, the game draws on a report by journalist Lucie Soullier in Le Monde (2015) that 
utilized an interactive presentation to convey the WhatsApp correspondence of a young Syrian, Dana, in her attempt to find refuge in Germany. Neither Majd nor Nour are real people so much as amalgamations of many tales told by those who, like Dana, have made the crossing being depicted:

Our two main characters, Nour and Majd, are fictional. They do not exist, or rather, they exist collectively. They are a multitude of men, women and children: Dana, her mother, her brother-in-law... as well as thousands of others who flee their country - or watch their relatives flee - all in hopes of finding a better life in Europe.

$B M M L$ thus synchronises multiple stories under the guise of a social media app rather than an overtly game-like aesthetic. In this way, the RIG highlights one of the most significant synchronies that arise in digital games: that is, the feel of 'gaming' itself - what Boluk and le Mieux term 'the standard metagame' (2017).

The standard metagame is the set of tropes, customs, technical standards, industrial structures and other shared elements through which digital games are coded primarily as part of consumerist 'geek culture'. Games are often evaluated with this set of values tacitly or overtly in the background: those which fail to provide sufficient diachronic excitement and thrills through technical overstimulation are maligned as 'not-games' or 'walking simulators' (Keogh 2018), reflective of a normative 'technicity' (Dovey \& Kennedy 2007). In the terms developed here, we can say that the unstable signifiers through which blockbuster games structure temporality are curiously stable: similar genres, similar characters, similar interfaces, similar themes, and so on. While there are certainly outliers and experimental works in gaming, there is a strong inertia that informs mainstream game development and design decisions, and directs the allocation of production resources at a high level.

On the other hand, games dealing with 'serious' subject matter can suffer from the low expectations that accompany playful activity and experience in the wider culture. The 'magic circle' of play becomes a synchronising meta-effect in which players are aware that what they are experiencing is 'just a game': an effect which Frank (2011) terms 'gamer mode'. This potentially leavens how players 
value the in-game representations and simulations, making an effective political freighting of the work difficult to achieve when compared to cinematic or literary works. This high-level gaming synchrony can thus act as an inertial drag on the cultural or political valuation of inventive digital games, such as $B M M L$, that depart from genre norms and make use of alternative aesthetic techniques.

At one level, the game design seeks to push back against the synchronising effects of the 'standard metagame' and thus re-introduce a sense of diachrony that seeks to convey the precarity of seeking refuge. This works through two main techniques: firstly, through submerging the game-like unstable signifiers (that is, the variables that determine diachronic possibilities in the narrative) beneath Nour's character and the social media interactions such as short messaging, photographs and emoticons. In a conventional design, these may be represented through bars or numerical values but in $B M M L$ a sense of their instability is retained. Secondly, the pseudo-real time progression of the game and incorporation of the game's icons into the typical social media interface elements of the mobile phone.

The depiction of Nour's journey and her interactions with Majd draws its inspiration from techniques of mobile and locative gaming more generally, but dissembles these through a resemblance to the temporal rhythms of smartphone usage. This allows the game to push back against the high-level synchrony of the standard metagame. The game does not take place within a neatly delimited magic circle, with players seated before the typical paraphernalia (mouse-keyboard-monitor or controller-TV set) that are emblematic of 'gaming'. Even as a smartphone app, BMML in its pseudo real-time mode emulates the piecemeal on-again-off again experience of chat rather than an 'immersive' game: the interface elements are cursory texts, emoji, selfies, and stresses about finding a power point, none of which are ever clear on their gameplay functionality. In the terms proposed by Zagal \& Mateas, $B M M L$ seeks to synchronise the frames of Gameworld Time, Coordination Time and Fictive Time, in order to leverage the confusion of temporal levels for aesthetic effect. 
diachrony that is further reflected in Nour's determined personality and the possibility that she will lie to Majd or disregard Majd's advice as player-character. This works against players' ability to build narrative anticipation, and without ascribing her the superhuman powers of many videogame protagonists, maintains a certain level of diachrony in the way that players relate to her future. In one of my playthroughs, Nour refused to don a veil in a Muslim country even though, given the mores of the area, it may have been safer for her to do so. Sometimes she will message saying that she is about to do something dangerous, or admit that she lied to Majd because she knew how he would react to her plans.

Nour's behaviour is responsive four variables: her morale, relationship level with Majd, her current budget and objects in her inventory. As noted, these are never displayed in-game as variables (apart from objects she may have collected), and instead are manifested in various ways through Nour's behavior. Where many story-based games will utilise a signifier to indicate when important choices are made (such as the 'butterfly-effect' motifs that indicates diachrony in Life is Strange (DontNod 2015)), in $B M M L$ there is no such indicator. Through the smartphone-chat interface and the submerged mechanics, $B M M L$ then preserves a powerful sense of the instability of its signifiers. This is a technique through which the game seeks to more effectively synchronise with the testimony of Dana in Soucille's Le Monde story than with the 'standard metagame' or 'gamer mode'.

The second powerfully diachronic aspect to $B M M L$ is the pseudo-real-time structure which allows the game to diachronically progress in a way that is closely linked to what smartphone users are by now very familiar with: the notification. Nour's attempts to contact Majd weave into the tasks, communications and updates of daily life, appearing as part of contemporary hypermodern time, rather than as a playful departure therefrom. They thereby become part of the harried consciousness a constant diachronic din - of the smartphone user. Often when she is implicated in a task or situation, Nour switches to 'busy' mode and no interaction is possible. The jostling stimuli of the smartphone thus become part of the game's 'paratext' (Consalvo 2007), helping to convey the anxiety that Majd 
(as representative of those left behind with a slender internet connection to a journeying loved one) must be feeling.

The forcefulness of this pseudo-real-time design can be compared with the experience of playing the game in 'fast mode' where there are no pauses between Nour's messages: the feedback loops of the game are immediately conveyed to players. In this mode, the magic circle and the 'gaminess' of the experience are far more evident: Nour's status as a game character is far more obvious, her capabilities bounded by the usual techniques of game design (branching paths, dialogue trees, and so on). It is when her messages catch players at different times and in different moods that the proper effect of the game arises. Even with the fast mode enabled, the game design is quite capacious, with 19 endings, multiple locales and choice points - it takes a considerable effort to chase them all down. This possibility space is compounded by the real-time mode - under the real-time game mode, players can't easily play through to find every end to the story. The tendency of replay to generate synchrony is resisted, thus tending to maintain the sense of diachrony.

Analysis of this unique game shows the capability of chronotypology to describe complex temporal schemes. In dissembling its interface through social media aesthetics, Nour's willful characterization and the pseudo-real-time progression, $B M M L$ staves off the most synchronic state in which players look back over a field of unstable signifiers which have been given definitive assignations and clearly represent the 'will-always-be-played'. Instead, the design seeks to maintain itself in the realm of the highly diachronic 'will-be-played' and the diachronic-synchronic mix of the 'can-be-played-with'. While maintaining diachrony in the individual play experience, $B M M L$ also synchronises many refugee stories from reality through its design, including that of Dana and the social media component through which her journey was rendered in Le Monde. It is in the interlacing of these temporal effects that the game seeks to recognize the value of lives lived in exile.

$B M M L$ won game industry coverage from major gaming press, and awards such as the Google Play Indie Games Contest 2017. This means that in spite of its tactical deferral of the standard metagame, it 
was recognizable enough to be recouped at another level of game culture. However, this perhaps points to its limitations as political speech, and once again we should heed Agamben's diagnosis that diachrony and synchrony are liable to flip over into one another.

Figure 1.

As we can see from this Tweet (Zenazir, online), gaming itself is something of an unstable signifier and many can be horrified at the coincidence of play and serious subject matter. Introducing the diachrony of play into what is perceived by some as synchronic historical fact runs the risk of trivialization. In this light, $B M M L$ shows that the value of subverting the standard metagame is, perhaps, mostly apparent to those who already know what that game is.

This discussion has demonstrated the potential of chronotypology to contribute nuanced analysis to temporal frames and heterochronia in gaming. Identifying unstable signifiers and conducting comparative analysis of diachrony and synchrony can assist scholars in posing and answering the sequence questions that arise in digital games. This includes a versatile definition of narrative as a highly synchronizing element that establishes a certain relation to the 'end of the game': an approach to game narrative that is neither better nor worse than those of formalism or ontology, just different.

\section{REFERENCES}

Aarseth, E.J., 1997. Cybertext: Perspectives on Ergodic Literature. The Johns Hopkins University Press, Baltimore.

Aarseth, E., 2004. Genre Trouble: Narrativism and the Art of Simulation, in: Wardrip-Fruin, N., Harrigan, P. (Eds.), First Person: New Media as Story, Performance and Game. The MIT Press, Cambridge, MA.

Agamben, G., 1993. Infancy and History: essays on the Destruction of Experience. Verso, London. 
Apperley, T., 2010. Global Rhythms: Play and Counterplay from the Situated to the Global. Institute of Network Cultures, Amsterdam.

Author 1., 2017. Peformativity in Art, Literature and Videogames. Palgrave Macmillan, London.

Bithell, M. and Burns, M., 2015. The Writer Will Do Something. Online resource:

https://mrwasteland.itch.io/twwds. Retrieved 05/02/2019.

Bloom, G., 2018. Gaming the Stage: Playable Media and the Rise of English Commercial Theatre. University of Michigan Press, Ann Arbor.

Boluk, S. and LeMieux, P., 2017. Metagaming: Playing, Competing, Spectating, Cheating, Trading, Making, and Breaking Videogames. University of Minnesota Press, Minneapolis.

Capcom, 2019. Resident Evil 2.

Despain, W. (ed)., 2008. Professional Techniques for Video Game Writing. AK Peters, Wellesley, MA.

Dominguez, C., Saussy, H. and Villanueva, D., Introducing Comparative Literature. Routledge, New York, NY.

Dovey, J. and Kennedy, H., 2006. Game Cultures: Computer Games as New Media. Open University Press, Berkshire.

Epic Games, 2018. Fortnite. 
Frasca, G., 2003. Simulation versus Narrative: Introduction to Ludology, in: Wolf, M.J.P.\& P. (Ed.), The Video Game Theory Reader. Routledge, London.

Ford, M., 2016. Writing Interactive Fiction With Twine: Play Inside a Story. Que, Indianapolis, IA. Frank, A. 2011. Gaming the Game: A Study of the Gamer Mode in Educational Wargaming. Simulation \& Gaming 43(1), 118-132.

The Fullbright Company, 2013. Gone Home.

Heussner, T., Finley, T., Hepler, J. and Lemay, A., 2015. The Game Narrative Toolbox. Focal Press, Burlington, MA.

Jauss, H.R. and Benzinger, E., 1970. Literary History as a Challenge to Literary Theory. New Literary History 2(1), 7-37.

Keogh, Brendan., 2018. A Play of Bodies: How We Perceive Videogames. The MIT Press, Cambridge, MA.

Koenitz, H., 2018. What Game Narrative Are We Talking About? An Ontological Mapping of the Foundational Canon of Interactive Narrative Forms. Arts, 7(4), 51.

Kryswinska, T., 2015. The Gamification of Gothic Coordinates in Videogames. Revenant $1.1,58-78$.

Lipovetsky, G., 2005. Hypermodern Times. Polity, Cambridge, MA. 
Machine Games, 2017. Wolfenstein II: The New Colossus.

McMillan, R., 2018. Reality-Inspired Games, personal correspondence.

Nikolchina, M., 2017. Time in Video Games: Repetitions of the New. Differences: A Journal of Feminist Cultural Studies, 28(5), 20-43.

The Pixel Hunt, 2017. Bury Me, My Love.

Ryan, M., 2014. "Emotional and Strategic Conceptions of Space in Digital Narratives", in Interactive Digital Narrative: History, Theory and Practice. Ed. Koenitz, H; Ferri, G; Haahr, M; Sezen, D; Tonguc, I. Routledge, New York, NY, 106-120.

Skolnick, E., 2014. Video Game Storytelling: What every developer needs to know about narrative techniques. Watson-Guptill, New York, NY.

Sony Santa Monica, 2018. God of War.

Soullier, L., 2015. “Le Voyage D’Une Migrante Syrienne A Travers Son Fil Whatsapp”, Le Monde. Online resource: https://www.lemonde.fr/international/visuel/2015/12/18/dans-le-telephone-d-unemigrante-syrienne_4834834_3210.html\#/. Retrieved 05/01/2019.

Zagal, J. and Mateas, M. Time in Video Games: A Survey and Analysis. Simulation Gaming, 41(6), $1-25$.

https://twitter.com/zenazir/status/1021352863308681216 
